\title{
Espaces, ouvertures et organes de circulation en hauteur dans l'église romane
}

Table ronde organisée par le CESCM de Poitiers et le CEM d'Auxerre (Auxerre, CEM Saint-Germain, 16 et 17 mai 2014)

\section{Sébastien Biay and Annick Gagné}

\author{
(2) OpenEdition \\ 1 Journals \\ Electronic version \\ URL: https://journals.openedition.org/cem/13889 \\ DOI: $10.4000 /$ cem.13889 \\ ISSN: 1954-3093 \\ Publisher \\ Centre d'études médiévales Saint-Germain d'Auxerre
}

\section{Electronic reference}

Sébastien Biay and Annick Gagné, "Espaces, ouvertures et organes de circulation en hauteur dans l'église romane", Bulletin du centre d'études médiévales d'Auxerre | BUCEMA [Online], 19.1 | 2015, Online since 11 June 2015, connection on 22 September 2022. URL: http://journals.openedition.org/cem/ 13889 ; DOI: https://doi.org/10.4000/cem.13889

This text was automatically generated on 22 September 2022.

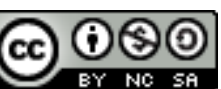

Creative Commons - Attribution-NonCommercial-ShareAlike 4.0 International - CC BY-NC-SA 4.0 https://creativecommons.org/licenses/by-nc-sa/4.0/ 


\title{
Espaces, ouvertures et organes de circulation en hauteur dans l'église romane
}

\author{
Table ronde organisée par le CESCM de Poitiers et le CEM d'Auxerre \\ (Auxerre, CEM Saint-Germain, 16 et 17 mai 2014)
}

\section{Sébastien Biay and Annick Gagné}

Lorsque, à la fin du $\mathrm{xII}^{\mathrm{e}}$ siècle, le moine Gervase entreprend de décrire l'ancienne cathédrale de Cantorbéry - l'église de Lanfranc, qui venait d'être la proie des flammes -, il commence par la tour de croisée, "placée au milieu de l'église comme au centre d'un cercle. Elle possède un ange doré à son sommet. À l'ouest est la nef ou aula, supportée de chaque côté par huit piles.» De cette nef, il pose

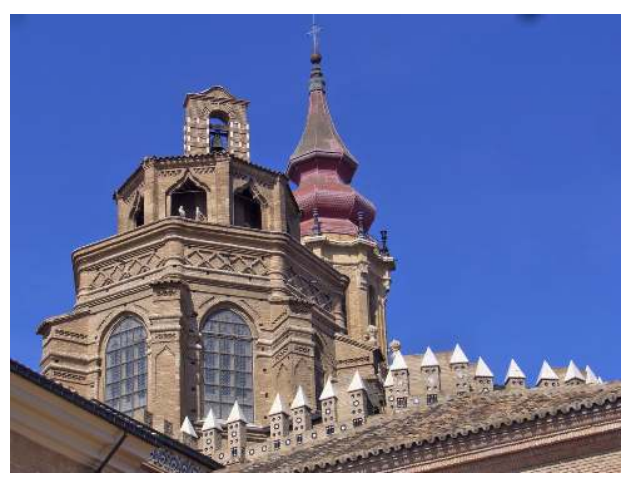
aussitôt les bornes: «Deux hautes tours pourvues de pointes dorées. » Puis, développant la description de l'édifice, il ne manque jamais de mettre en relation le bas avec le haut, notamment pour les chapelles superposées des bras du transept, ni d'indiquer l'emplacement des degrés ou des marches par lesquels on descend dans la crypte ou l'on s'élève vers le sanctuaire ${ }^{1}$.

Depuis quelques années, l'étude des églises médiévales donne une attention accrue aux rapports entre les formes architecturales et les fonctions rituelles. L'enquête doit être menée dans les trois dimensions de l'espace. Or, la dimension de la hauteur, dont l'importance se manifeste en particulier dans le récit de Gervase, par la mise en avant des tours et les précisions qu'il apporte dans les relations verticales et les changements de niveau, ne semble pas avoir encore reçu une attention suffisante ${ }^{2}$. Quelques dossiers ont bénéficié d'un éclairage spécifique : les massifs et « chœurs-halles » occidentaux ${ }^{3}$, les tribunes destinées aux femmes ${ }^{4}$, les tours mettant en valeur un hôte de marque ${ }^{5}$, 
les chapelles privées ${ }^{6}$. Les actes du colloque Avant-nefs et espaces d'accueil ont fait apparaître des carences en données archéologiques et de nombreuses difficultés d'interprétation des salles hautes dans cette partie singulière de l'église. Elles ont résonné, pour les initiateurs de cette table ronde, comme un appel à la réouverture d'un chantier. Ce chantier ne se limite pas aux seuls problèmes posés par les parties hautes des espaces d'accueil, mais entend aborder l'ensemble des formes et des dispositifs architecturaux situés dans les étages supérieurs des églises romanes. Nous avons en effet souhaité concentrer l'enquête sur « l'époque romane ». La cohérence que l'on ambitionne de préserver de cette manière n'est-elle pas quelque peu surfaite? C'est probable. Dès lors qu'on ne cherche pas à saisir des moments particuliers ou charnières, si ce n'est de manière locale (Aquitaine, Bourgogne), l'ouverture chronologique ne semble pas plus dommageable à la cohérence du discours que l'ouverture géographique. Ainsi les édifices présentés comptent parmi eux nombre d'avatars de ce que l'on désigne habituellement comme "style roman tardif», voire très tardif... Il convient surtout de prévenir toute mise en série abusive, de ne pas tenter de faire correspondre de manière unilatérale des formes, des fonctions et des contenus symboliques. Il s'agit plutôt de partir en quête de nouveaux indices, de croiser les réflexions sur les choix formels et les transformations des édifices avec les exigences liturgiques et les conditions pratiques de leur mise en œuvre, sans négliger le discours symbolique sur le lieu de culte ${ }^{7}$.

3 Ainsi ont été réunis dans les murs du Centre d'études médiévales Saint-Germain d'Auxerre (CEM), les 16 et 17 mai 2014, et grâce au soutien du CEM et du Centre d'études supérieures de civilisation médiévale (CESCM, université de Poitiers/CNRS), des chercheurs concernés par ces questions et représentant les champs de spécialisation de l'archéologie monumentale, de l'histoire de l'art, de la liturgie et de l'histoire des textes. Les organisateurs de cette table ronde à huis clos tiennent à remercier chaleureusement tous les contributeurs ${ }^{8}$, auteurs de communication ou intervenants des discussions: Claude Andrault-Schmitt, Sylvain Aumard, Tancredi Bella, Stéphane Büttner, Eduardo Carrero Santamaría, Pascale Chevalier, Fabrice Henrion, Clemens Kosch, Arlette Maquet, Alain Rauwel, Christian Sapin et Cécile Treffort ${ }^{9}$.

\section{Accéder aux espaces hauts : circulations verticales dans l'église}

4 La condition de l'élévation des clercs comme des laïcs dans l'église médiévale est la construction d'organes de circulation entre le haut et le bas. Christian Sapin a présenté les escaliers tournants de Saint-Bénigne de Dijon. Deux escaliers à vis mentionnés dans la chronique du XI siècle flanquent la rotonde au nord et au sud ${ }^{10}$. Celui côté sud, d'une largeur de 1,20 m, est doté d'un puits central. De plus, l'iconographie ancienne de la rotonde, en grande partie ruinée, atteste la présence d'une tourelle percée de baies dans la partie nord, suggérant la possibilité d'un éclairage zénithal de l'escalier ${ }^{11}$. Quasi contemporain de l'escalier de Saint-Bénigne, celui de l'avant-nef de Saint-Vincent de Mâcon est lui aussi d'une largeur conséquente mais ne possède pas de noyau central. Le chantier de Saint-Bénigne montre ainsi une forte invention dans la mise en œuvre des escaliers. Il est vraisemblable que la communauté en totalité ou représentée par des groupes empruntait cet escalier de façon régulière, la circulation entre le haut et le bas 
de l'église étant très fréquente dans la vie monastique clunisienne. Quelle quantité de lumière souhaitait-on diffuser à l'intérieur de cet escalier? Quelle que soit la réponse, on imagine que la suspension de luminaires dans le puits central aurait pu compléter, si le besoin s'en était ressenti, l'éclairage zénithal naturel ${ }^{12}$.

L'examen des accès aux étages est d'une grande importance pour une compréhension fonctionnelle de ces espaces. Tancredi Bella en a fait la démonstration à propos de l'église Saint-Ambroise à Milan - progressivement rebâtie entre le XI ${ }^{e}$ et le XII siècle -, dont les tribunes ont généralement été envisagées par les historiens de l'art dans leur seule fonction architectonique (fig. 1) - une fonction bien assurée, dont les croquis avant restauration de l'architecte Fernand de Dartein (1838-1912) permettent de comprendre le fonctionnement et dont $\mathrm{M}$. Bella a pris la peine d'expliquer le détail ${ }^{13}$ ou comme une citation de l'architecture paléochrétienne (on pense aux fausses tribunes de la cathédrale de Modène).

Fig. 1 - Milan, Sant'Ambrogio, vue des tribunes depuis l'ouest (cl. T. Bella).

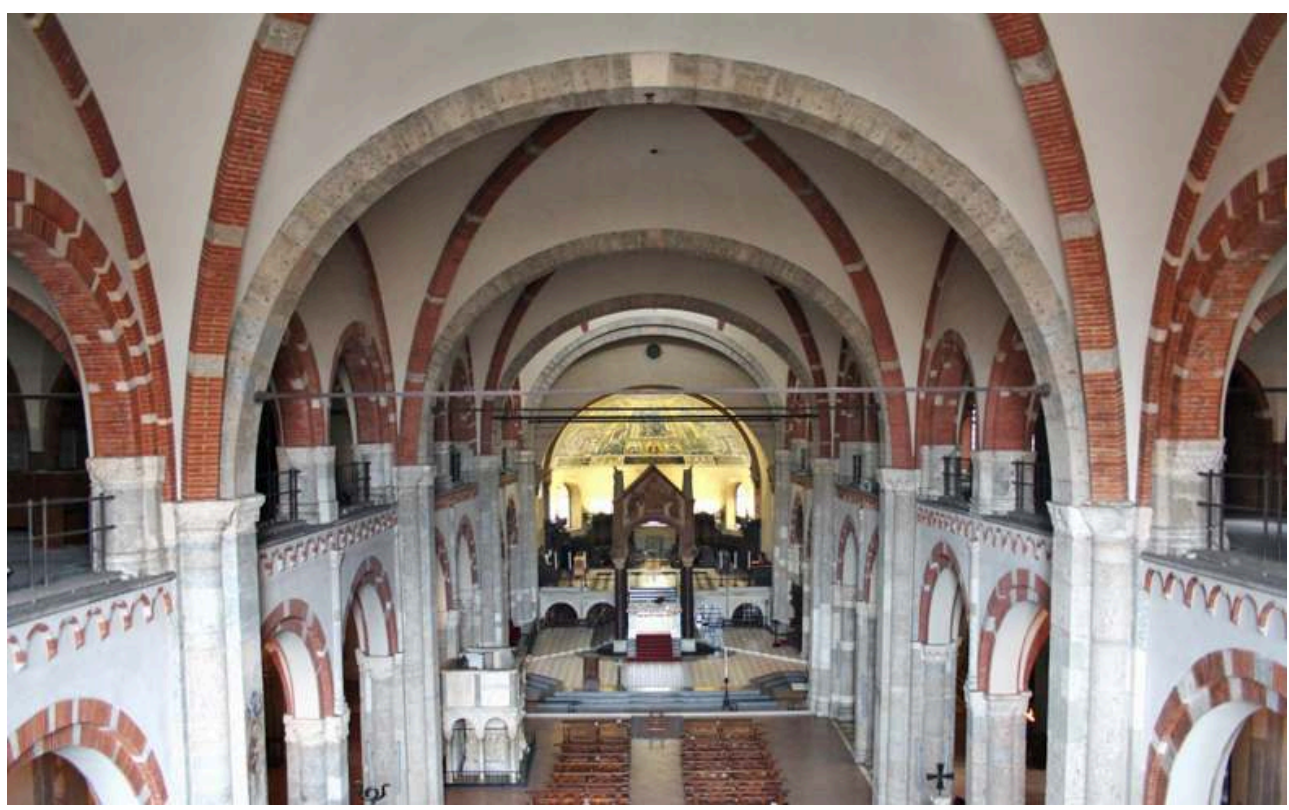

Or, il est vraisemblable que les restaurations successives de l'église ont fait disparaître les traces archéologiques d'un possible usage liturgique de ces tribunes, et la question de l'accès - qui se fait aujourd'hui par une sacristie - mérite une attention particulière. À l'époque romane, il devait se faire par les deux tours de façade, qui étaient peut-être raccordées l'une à l'autre par une haute tribune extérieure au-dessus du porche. En 1128, l'archevêque Anselme V fit bâtir un nouveau campanile (l'actuelle tour nord) et le donna à la communauté des chanoines; ceux-ci desservaient l'église, tandis que l'abbaye observait une règle monastique - bipolarité à la source de conflits entre les religieux ${ }^{14}$. Chaque communauté pouvait ainsi accéder aux tribunes par sa tour et prendre part à la liturgie. On sait que deux liturgies étaient pratiquées alternativement à Saint-Ambroise; la question est posée d'une véritable liturgie commune où les tribunes auraient joué un rôle important. 


\section{Personnalisation et fréquentation des lieux : de la difficulté d'attribuer une destination précise}

7 Le dossier de Saint-Ambroise soulève le problème de la destination de ces espaces élevés à une communauté, d'une partie de communauté ou à des personnalités particulières, problème qui concerne également le dispositif singulier de l'église de Saint-Aubin en Côte-d'Or (Christian Sapin), qui fera l'objet de prochains travaux du CEM. Un oratorium fut donné par le duc de Bourgogne aux chanoines de Beaune au $\mathrm{x}^{\mathrm{e}}$ siècle. De ce siècle datent les murs de la nef (datation ${ }^{14} \mathrm{C}$ ). La partie orientale et la partie occidentale de l'église possèdent des tribunes largement ouvertes sur la nef (fig. 2).

Fig. 2 - Saint-Aubin, coupe longitudinale de l'église (CEM).

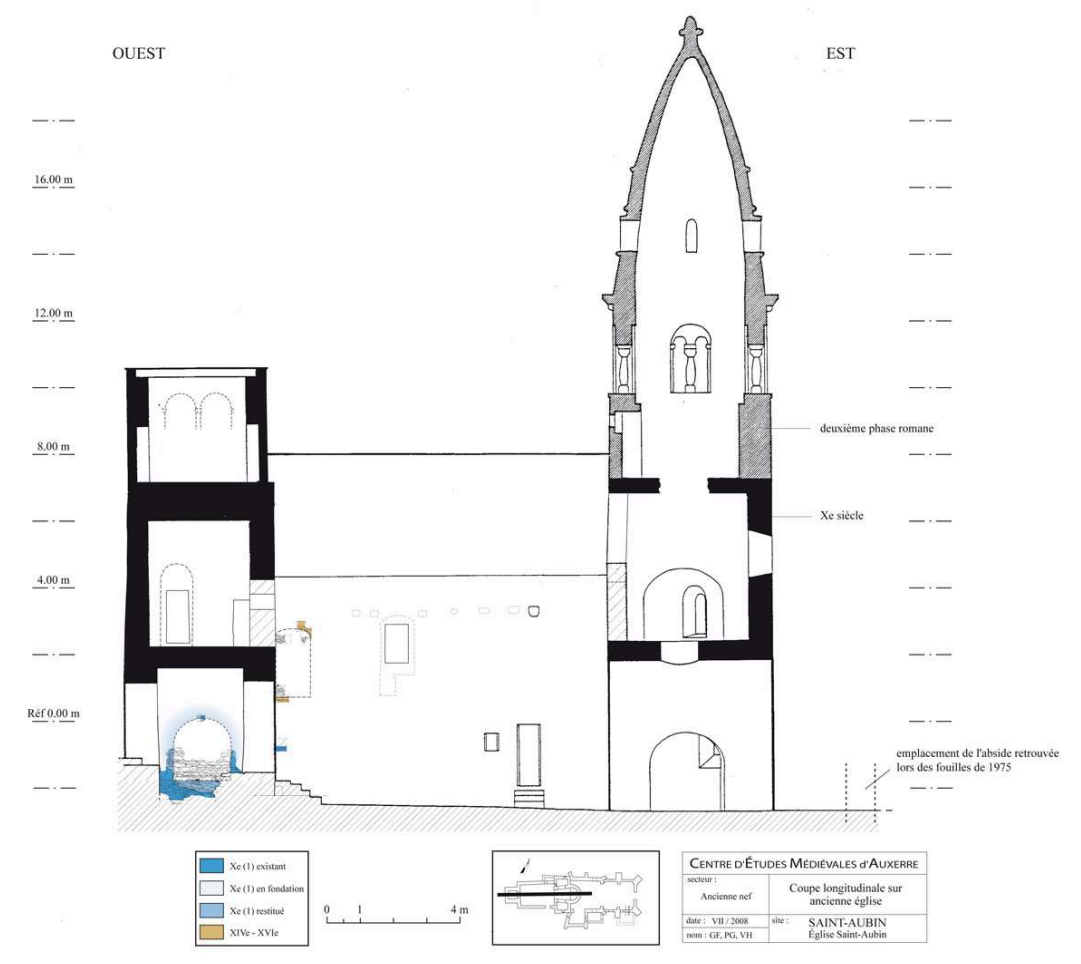

Elles se regardent à une hauteur équivalente. Ce dispositif n'est pas sans rappeler l'architecture des chapelles palatines - y compris par ses dimensions réduites -, mais son interprétation reste largement ouverte. Comment les deux espaces fonctionnaientils ensemble? Tout le problème est là ! L'église recèle un indice supplémentaire. Une fenêtre à coussièges percée dans le mur septentrional de la nef au xiII siècle et aménagée à l'extérieur de l'église regarde en direction de l'étage occidental et semble insister sur l'importance de celui-ci. Ce dossier soulève la question de la place accordée aux grands seigneurs, aux puissances telles que les chanoines, abbés ou prieurs, ainsi qu'à une éventuelle division des sexes organisée par un dispositif séparant le haut et le bas. L'église de Saint-Aubin évoque une résidence privilégiée, que pourtant aucun acte ne documente contrairement à d'autres exemples régionaux. La baie à coussièges du $\mathrm{XIII}^{\mathrm{e}}$ siècle pose également la question des contacts visuels entre les parties d'un édifice 
- ou entre plusieurs corps de bâtiment -, que nous retrouverons à propos de la cathédrale de Naumbourg.

Tout aussi problématique est le dispositif interne de l'église Santa Maria du Tiglio à Gravedona (près de Côme) présenté par Tancredi Bella. Probablement édifiée sur les restes d'un baptistère paléochrétien du $\mathrm{v}^{\mathrm{e}}$ siècle dédié à Saint-Jean-Baptiste, l'église Sainte-Marie était déjà fonctionnelle à l'époque carolingienne. La reconstruction romane, au début de XII ${ }^{\mathrm{e}}$ siècle, est forcément antérieure à 1154, année où l'église SaintVincent, toute proche, subit un écroulement de sa partie orientale et un transfert temporaire du chapitre à Sainte-Marie. L'espace intérieur est de plan centré à nef unique, une abside principale orientée et deux absides latérales. Deux galeries scandées par de fines colonnes à chapiteaux sculptés courent dans la partie haute des murs latéraux; on y accède par deux escaliers à vis aménagés dans le mur occidental. Si l'on peut imaginer un parcours d'un escalier à l'autre, il est tout aussi vraisemblable qu'escaliers et galeries aient eu vocation à distinguer deux groupes. La fonction de l'église était paroissiale; elle n'était donc pas destinée à accueillir une communauté religieuse, mais pouvait être sollicitée pour des solennités particulières. En l'absence de données précises sur les relations entre Sainte-Marie et l'église Saint-Vincent, la distribution des services liturgiques de l'une et de l'autre, le problème du rôle de ces galeries reste entier ${ }^{15}$.

Sylvain Aumard a mis en évidence sur deux sites bourguignons et champenois l'existence de galeries de bois dont la destination est tout aussi problématique. Il s'agit des églises de Brienne-la-Vieille (Aube, $\mathrm{x}^{\mathrm{e}}$ siècle) et Laroche-Saint-Cydroine (Yonne, $\mathrm{XI}^{\mathrm{e}}$ siècle). Ces deux églises possèdent des charpentes à voûtes lambrissées en bois. La présence de mortaises destinées à fixer des poteaux verticaux dépourvus de fonction structurelle permet de restituer des galeries de circulation intérieures sur les flancs de la nef. Des éléments de comparaison existent dans l'architecture protestante, ou encore dans l'architecture juive, où le dispositif permet une séparation des hommes et des femmes. Les systèmes de galeries sont par ailleurs fréquents dans l'architecture laïque et pourraient l'être beaucoup plus répandus qu'on ne le pense généralement ${ }^{16}$ - à se demander si les deux salles hautes de l'église de Saint-Aubin ne pouvaient pas être reliées par des galeries de ce genre. A-t-on éprouvé le besoin d'augmenter la capacité d'accueil des églises en question? Ou s'agirait-il d'un système utilisé ponctuellement, pour des solennités (Noël, Rameaux)?

\section{Construire la mémoire d'un lieu}

La destination des espaces hauts, envisagée dans les exemples précédents du point de vue des modalités d'accès et de la distinction de groupes ou de personnes dans des lieux propres, se pose aussi naturellement dans les termes d'une interaction entre le haut et le bas dans le cadre de la liturgie eucharistique (on y viendra) comme dans celui de la construction de la mémoire et de la mise en scène de monuments funéraires, ce qu'illustre en particulier l'église Saint-Vorles de Marcenay (Christian Sapin), lieu d'origine des reliques de saint Vorles qui furent transférées à Châtillon-sur-Seine au $\mathrm{IX}^{\mathrm{e}}$ siècle. L'église actuelle a conservé dans ses murs des traces (reprises en sous-œuvre) d'un édifice important de la fin du $\mathrm{IX}^{\mathrm{e}}$-début $\mathrm{du} \mathrm{X}^{\mathrm{e}}$ siècle. L'observation d'indices dans l'aménagement de ces lieux permet de dégager un faisceau de problèmes. En arrière du 
clocher du XVIII ${ }^{e}$ siècle et au-dessus de la première travée de la nef existe un étage auquel on ne peut désormais accéder que par une tourelle tardive (fig. 3).

Fig. 3 - Marcenay, Saint-Vorles, coupe longitudinale (CEM).

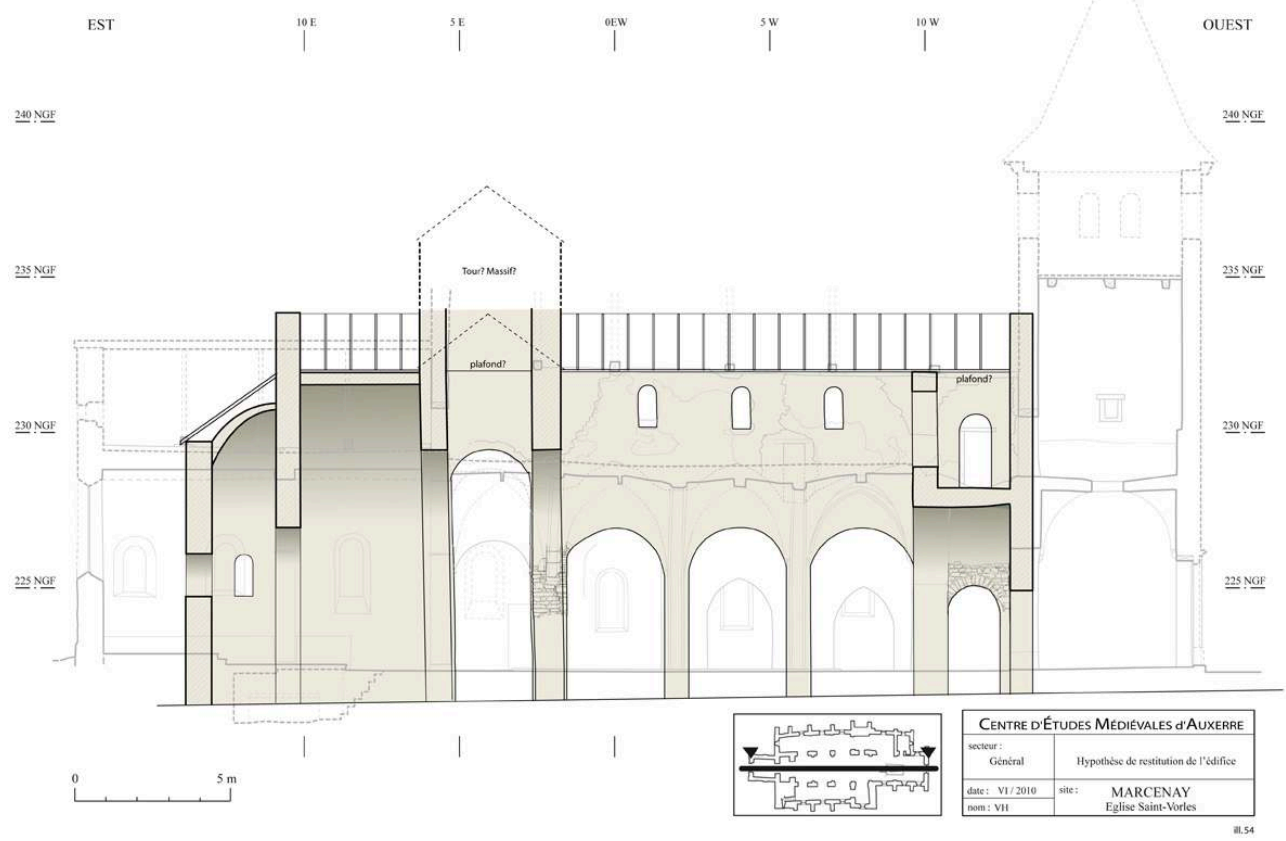

12 On ne conserve pas de trace de sol dans cette partie de l'église, sinon un petit massif situé dans l'axe et qui pourrait avoir appartenu à un autel (fig. 4). 
Fig. 4 - Marcenay, Saint-Vorles, relevé au sol de l'étage de la nef (CEM).

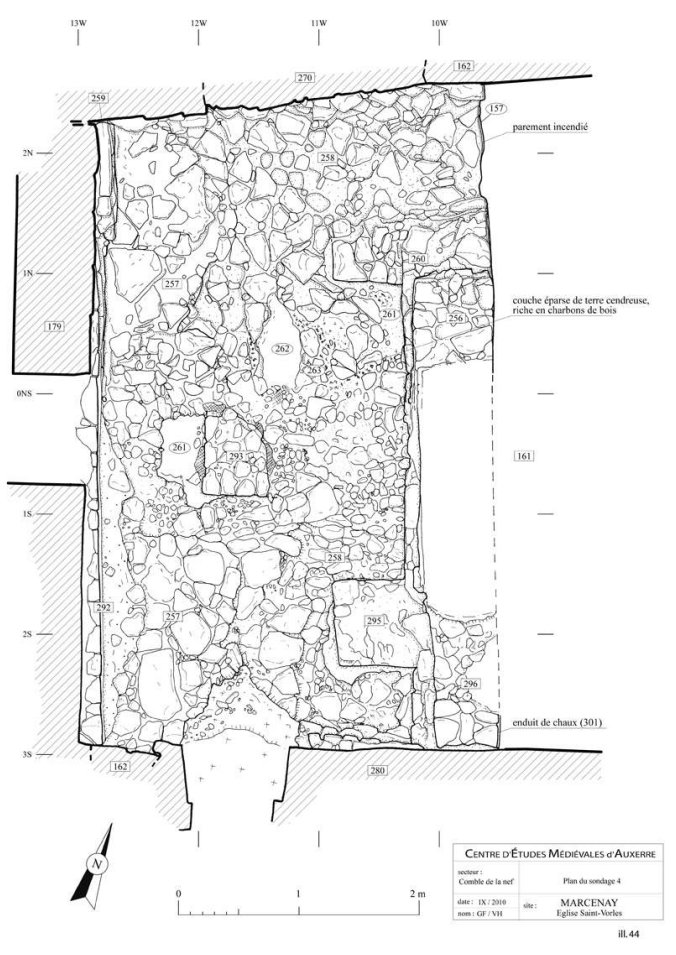
partie orientale de l'église - et que rien n'empêche de dater de l'époque du saint, le $\mathrm{VI}^{\mathrm{e}}$ siècle -, aurait pu être exposé dans l'abside. La possibilité d'un chevet relativement haut suggère qu'une communication visuelle était possible entre l'étage occidental et le sarcophage. On sait par ailleurs que le massif occidental de l'église de Châtillon-surSeine possédait un étage - avait-on sciemment repris le dispositif de Marcenay?

La mémoire des défunts concerne également le dossier épineux de l'ancienne église Saint-Pierre à Saint-Père près de Vézelay ${ }^{17}$. Le site est un lieu d'inhumation au moins depuis le $\mathrm{VI}^{\mathrm{e}}$ siècle. La charte de fondation du monastère féminin par Girart de Vienne ne dit rien des éventuelles structures antérieures. L'église, de petite dimension, possède un chevet du début de l'époque romane. Un massif occidental à étage, dont une photographie ancienne atteste qu'il était éclairé d'une fenêtre, a été construit sur une zone de sépulture. Il n'est pas sans évoquer les tours de Zadar ou de Cetina en Croatie ${ }^{18}$ L'église Saint-Pierre fut reconstruite après le transfert de la fondation religieuse sur la colline de Vézelay. Pourquoi a-t-on doté d'un massif occidental à étage un édifice aussi peu développé en longueur? Les inhumations situées sous le massif seraient-elles l'enjeu? La raison d'être de la nouvelle église Saint-Pierre (xIII ${ }^{\mathrm{e}}$ siècle) est encore plus problématique. Remplacerait-elle l'édifice principal du monastère carolingien, dont l'église romane serait une église secondaire? La tour de l'ancienne église Saint-Pierre faisant face à la colline de Vézelay, on peut s'interroger sur l'existence d'un lien visuel (depuis le haut de la tour) avec le monastère de la Madeleine, un peu à l'image de l'ensemble poitevin de Saint-Jouin-de-Marnes (Deux-Sèvres), qui associait monastère d'homme en partie haute du terrain, monastère de femme en partie basse et tour seigneuriale édifiée par le comte d'Anjou sur la colline en face. On n'a cependant 
aucune certitude quant à la continuité de la vie monastique sur le site après la fondation du monastère de Vézelay.

\section{Continuité et discontinuité des motifs dans le temps}

Les avant-nefs - terme englobant des réalités très différentes - occupent une place de choix dans le dossier des motifs architecturaux à étage en Bourgogne. À Auxerre, outre celle de Saint-Germain, dont aucune source ne permet de restituer la forme de l'espace haut, la cathédrale Saint-Étienne possédait également une partie occidentale développée : une tour bâtie à l'époque carolingienne, reprise au $\mathrm{x}^{\mathrm{e}}$ siècle et dont le programme pictural nous est connu ${ }^{19}$. La salle haute occidentale de Saint-Marcel à Saint-Marcel-lès-Chalon aurait quant à elle hérité de l'architecture ottonienne aux alentours de l'an Mil, antérieurement à l'émergence du «modèle clunisien » des galilées - si tant est qu'on puisse le désigner ainsi ${ }^{20}$.

L'avant-nef de l'abbatiale de Vézelay, qui appartient à cette série des galilées selon la thèse de Kristina Krüger ${ }^{21}$, a également fait l'objet d'une présentation détaillée. Stéphane Büttner a ainsi livré les observations et les questions issues de son travail sur le grand portail de la nef, la façade occidentale de la nef et l'avant-nef ${ }^{22}$. Un premier projet d'avant-nef avec un étage ouvert ou rythmé en façade par des baies aveugles a pu être mis en évidence. Ce premier projet s'étendait au moins sur la travée précédant les portails et il incluait les deux portails latéraux de la nef. Si le grand tympan correspond lui aussi à ce premier état, il est difficile d'affirmer la même chose pour l'ensemble du portail. Un rehaussement des voûtes et une surélévation de l'espace de circulation à l'étage correspondent à la mise en œuvre du second projet, qui a donné à l'avant-nef sa forme définitive. Le chantier de l'abbatiale de Vézelay se situe entre les années 1120 et 1150 ; le changement de projet fut donc contemporain de la construction de l'avant-nef de l'abbatiale de Cluny, dépourvu d'étage. L'avant-nef de Vézelay peut quant à elle être comparée à celle de la priorale de Perrecy-les-Forges ou celle de Châtel-Montagne. N'aurait-on pas repris une forme d'avant-nef ancienne, puis opéré une transformation en cours de chantier? Cette transformation traduirait-elle une réflexion sur la fonction respective des espaces inférieurs et supérieurs ou leur relation? Une question qui invite notamment à s'interroger sur la cohérence fonctionnelle de la série des galilées clunisiennes.

Dans les mêmes années (1120-1150), d'autres transformations, suggérant là aussi des changements dans la conception et l'utilisation des salles hautes, survinrent en Limousin, comme l'a évoqué Claude Andrault-Schmitt. Bien que les formes fussent toujours impressionnantes, la construction des clochers ne s'accompagna plus d'une division interne par la présence d'un étage - Le Dorat, Saint-Junien, La Souterraine, Bénévent ${ }^{23}$. Il faut attendre la fin $\mathrm{du} \mathrm{XII}^{\mathrm{e}}$ siècle pour voir renaître l'engouement pour les tours-porches aménagées de salles importantes (Solignac, Saint-Martin de Tulle).

\section{Les espaces latéraux : un domaine en friche}

18 Claude Andrault-Schmitt a également insisté sur la nécessité de prendre en considération les espaces latéraux de l'église, en particulier les transepts, souvent délaissés par la recherche. Les tours de transept de l'abbatiale de saint Hugues à Cluny 
ont à l'évidence inspiré le chantier de la cathédrale Saint-Pierre d'Angoulême. Le clocher le plus élevé, situé du côté de la falaise et financé par l'évêque, faisait de cette cathédrale la plus haute église de France jusqu'au XVI e siècle. Comme à Cluny, les espaces hauts du transept d'Angoulême sont évidés au profit de la lumière - vers 1175, les tribunes de la nef de Saint-Martin de Tours furent supprimées, on imagine dans le même esprit de ménager une grande hauteur sous voûte, un exemple qui a peut-être inspiré la cathédrale de Bourges.

Les chapelles latérales au chevet et autres sacristies à étage forment un ensemble riche et divers. Le chantier de la collégiale de Saint-Yrieix s'ouvrit dans les années 1181-1183 par la construction d'une chapelle à deux niveaux. L'espace supérieur, voûté dès l'origine, recelait le chartrier. Une chapelle semblable fut construite à la collégiale de Saint-Junien en 1223, dédiée à saint Martial et donnant accès à l'espace de circulation autour du sanctuaire ${ }^{24}$. Les salles hautes sont également présentes dans l'architecture des ordres austères. À Grandmont comme à l'Artige (avant 1198), les espaces latéraux au chevet étaient ou sont très fermés par rapport au vaisseau central. Dans le premier cas, une chapelle à deux étages formait le pendant de la chapelle funéraire du fondateur de l'ordre, Étienne de Muret, côté sud. Au prieuré de Comberoumal (Aveyron), la salle située au-dessus de la sacristie et jouxtant le dortoir devait être dévolue à l'abbé ${ }^{25}$.

Autre phénomène peu ou pas étudié, celui des tribunes de transept, qui concerne tout particulièrement le monde anglo-normand. La description par Gervase de l'église de Lanfranc à Cantorbéry en mentionne la présence ; elles sont également importantes dans les chapelles-tours de la nouvelle cathédrale, qui ne sont jamais prises en compte dans les études de l'édifice.

21 La cathédrale Sainte-Agathe de Catane, édifiée à la demande de Roger $\mathrm{I}^{\text {er }}$ de Hauteville entre 1089 et 1094, constitue un autre exemple original de compartimentage et d'interconnexion entre espaces latéraux et chevet (fig. 5). 
Fig. 5 - Catane, cathédrale, partie sud du transept vue depuis l'est (cl. T. Bella).

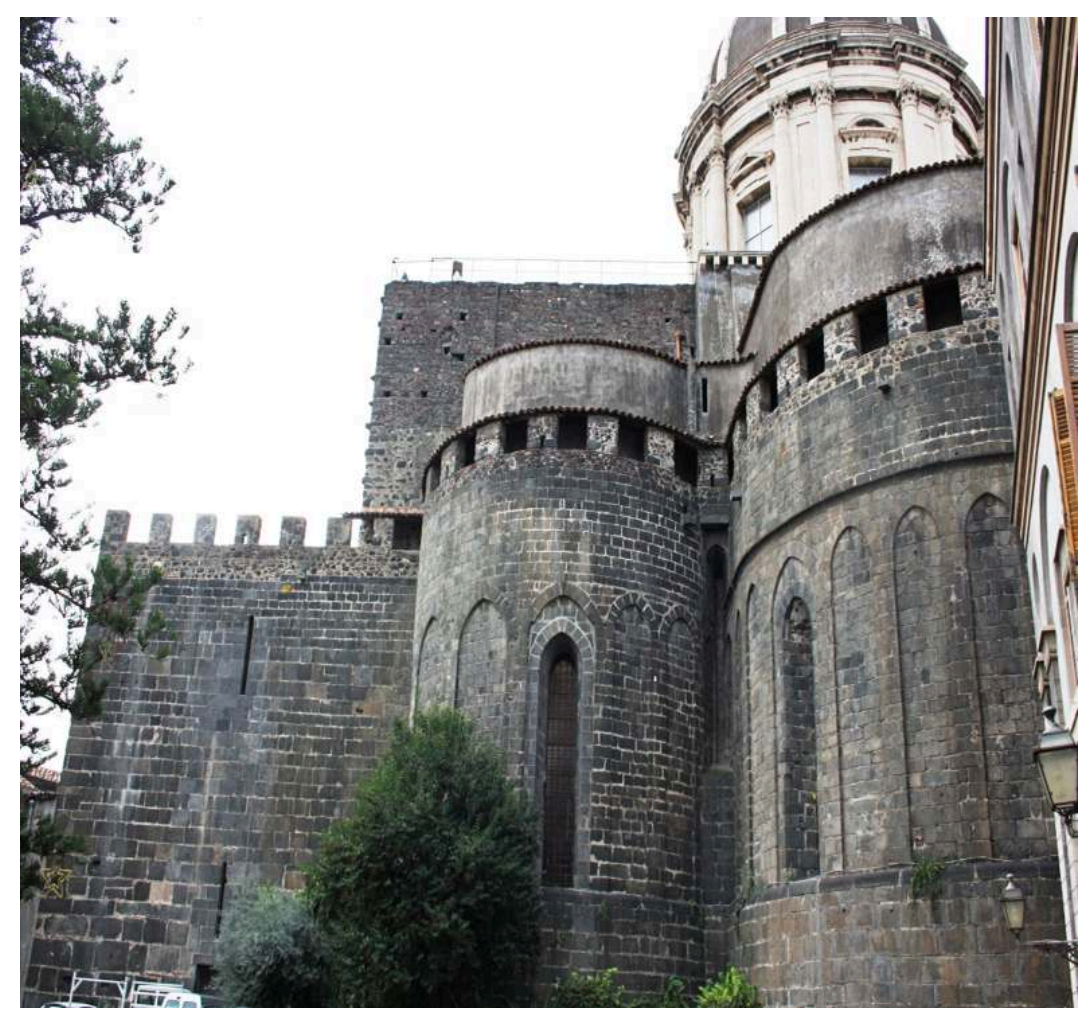

Originairement surélevé par rapport à la nef, le transept est refermé de chaque côté par deux compartiments latéraux à plusieurs niveaux. L'existence d'un chemin de ronde continu du transept au chevet confère une fonction de fortification à ces volumes latéraux auxquels on accédait par deux escaliers à vis dans les murs nord et sud - seul celui du nord est conservé ${ }^{26}$. Cela dit, les observations directes de Tancredi Bella ont permis de proposer l'hypothèse que les niveaux plus hauts étaient liés à une sorte de couloir ou de chemin haut qui courait dans l'épaisseur des murs entre les gouttereaux et le mur au-dessus des arcades longitudinales ou directement au-dessus des collatéraux comme une sorte de tribune. Quant à la tour du massif septentrional, qui mène à la salle haute, elle possède des fenêtres ouvertes en direction du presbyterium (espace de l'autel).

\section{Régler la liturgie, diffuser ses effets : de la nécessité des connexions sensorielles}

Ce contact visuel établi avec le presbyterium à la cathédrale de Catane suggère une interaction entre espace liturgique et espace haut, dont Clemens Kosch a étudié la configuration à la cathédrale de Naumbourg, allant souvent au-delà des données livrées par l'histoire des textes ${ }^{27}$.

24 La cathédrale de Naumbourg a été beaucoup étudiée depuis un siècle - les monographies ont fait la part belle aux statues polychromes du dit maître de Naumbourg dans l'abside occidentale. Il s'agit d'une cathédrale bipolaire, avec un chœur oriental (première moitié $\mathrm{du}$ XIII ${ }^{\mathrm{e}}$ siècle) et un chœur occidental (vers 1250) qui semblent équivalents en importance; on saisit encore mal aujourd'hui le 
fonctionnement de l'ensemble. L'examen attentif des systèmes d'accès au chœur occidental - qui conserve un jubé orné de sculptures et une tribune - permet de constater des circulations différentielles donnant accès au chœur - dont l'autel restait invisible aux laïcs - et de monter à la tribune d'une part, d'emprunter la coursière faisant le tour du chœur en arrière des fameuses statues d'autre part.

Des salles hautes sont aménagées dans les tours flanquant chacun des deux chœurs. La présence d'une niche dans la chapelle haute de la tour nord-ouest donne à réfléchir sur la localisation de la réserve eucharistique. À la suite du concile de Latran se diffuse l'idée que la réserve eucharistique doit se situer dans un endroit protégé des possibles agressions des hérétiques ${ }^{28}$. Cette chapelle donne une vue directe sur l'autel oriental de la croix. Il existe donc un lien visuel entre l'autel recevant les dévotions laïques et le lieu où auraient été conservées les hosties.

Les tours orientales sont dépourvues de chapelle. Les pièces intérieures sont néanmoins voûtées et devaient accueillir les éléments du trésor de la cathédrale. Un système de petits oculi met en rapport les niveaux superposés de la tour nord-est, ainsi que les pièces avec l'escalier. Toutes les parties de la tour sont ainsi liées entre elles de sorte que puisse y circuler la lumière, l'odeur de l'encens, le son, comme une diffusion du sacré par tous les pores de l'édifice et un moyen de prendre part à la liturgie d'où que l'on se trouve.

Au troisième niveau de la tour sud-est, une fenêtre s'ouvre en direction de l'ouest. Visible depuis les stalles les plus en avant du chœur oriental, elle l'était certainement du chantre - véritable coordonnateur de la liturgie - et devait lui permettre de communiquer visuellement - par le relai d'autres intervenants - avec les préposés aux cloches. Le chantre signalait ainsi de manière anticipée par rapport au déroulement de la liturgie le moment où les cloches devaient être mises en mouvement, afin qu'elles sonnent avant l'élévation de l'hostie.

L'étude de $\mathrm{M}$. Kosch est une invitation à s'interroger sur la mise en œuvre concrète de la liturgie. Les ressources textuelles ayant déjà été épuisées, c'est par l'observation des indices matériels et en s'interrogeant sur les installations nécessaires au bon fonctionnement de l'office divin, dont la réussite concrète est forcément une condition nécessaire de son efficacité, que peut être approfondie la connaissance des édifices. Cela doit être particulièrement vrai pour les cathédrales, qui incluent une communauté de fidèles et impliquent une mise en scène du corps ecclésiastique.

\section{Montrer, cacher : exposer et préserver les reliques}

Les communications visuelles dans le cadre de l'action liturgique rejoignent un autre aspect des relations entre le haut et le bas. En effet, les fidèles ont souvent levé la tête pour contempler des reliques montrées depuis une tribune, comme le voile de Véronique au Vatican, ou disposées sur des poutres de gloire comme à la cathédrale de Cantorbéry. Au XVII ${ }^{e}$ siècle, le jour de Pâques, le suaire de Besançon était montré aux fidèles massés à l'extérieur de la cathédrale depuis une galerie de bois ajoutée à l'ancien clocher occidental roman, comme l'a rappelé Alain Rauwel. Reconstruit au XVIII siècle, le clocher fut alors doté de balcons - reliés à une salle intérieure - pour faciliter l'ostension de la relique vers l'extérieur. Un système de galerie ouverte sur la ville a également existé à cette fin à la cathédrale d'Autun. 
30 La conservation des reliques dans des salles hautes est également bien attestée, exprimant le souci de placer les objets saints en lieu sûr - on l'a vu pour la conservation des hosties à la cathédrale de Naumbourg. C'est une hypothèse intéressante dès lors que les aménagements de ces espaces sont difficiles à interpréter. L'étage du clocherporche de l'église Santa Maria du Tiglio à Gravedona (fin XI ${ }^{\mathrm{e}}$-début XII ${ }^{\mathrm{e}}$ siècle), présentée par Tancredi Bella, pose ce genre de problème (fig. 6).

Fig. 6 - Gravedona, Santa Maria del Tiglio, vue du massif occidental depuis l'ouest (cl. T. Bella).

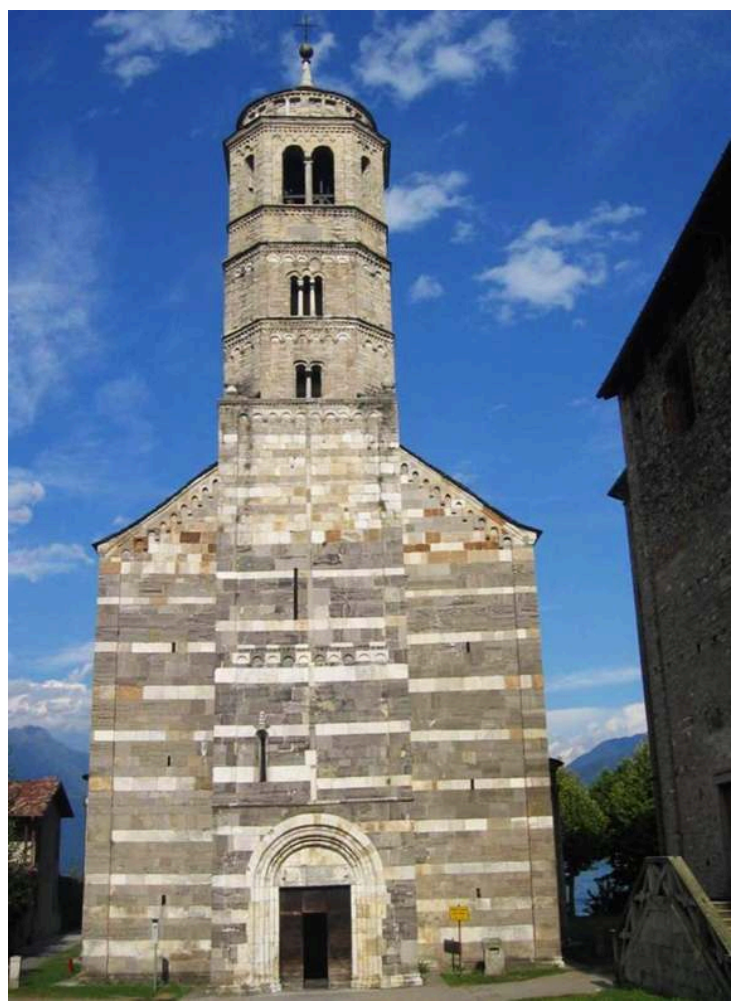

31 Il s'agit d'une chapelle voûtée en berceau, de construction soignée, partiellement visible depuis l'extérieur par une petite fenêtre et ouverte par une baie - au fond d'un petit passage mural - sur l'intérieur de l'église. Une niche, de 30 par $40 \mathrm{~cm}$ environ, aménagée dans le mur, aurait pu servir de placard à reliques ${ }^{29}$.

Il n'est guère possible de restituer avec certitude le contenu d'une niche ou d'une cavité, mais son emplacement peut légitimement soulever la question de la conservation des reliques. Celle de l'église Saint-Nizier à Accolay (milieu XII siècle), décrite par Fabrice Henrion, entre dans cette série ${ }^{30}$ (fig. 7). 
Fig. 7 - Accolay, Saint-Nizier, vue depuis la travée droite en direction de la travée sous clocher (CEM).

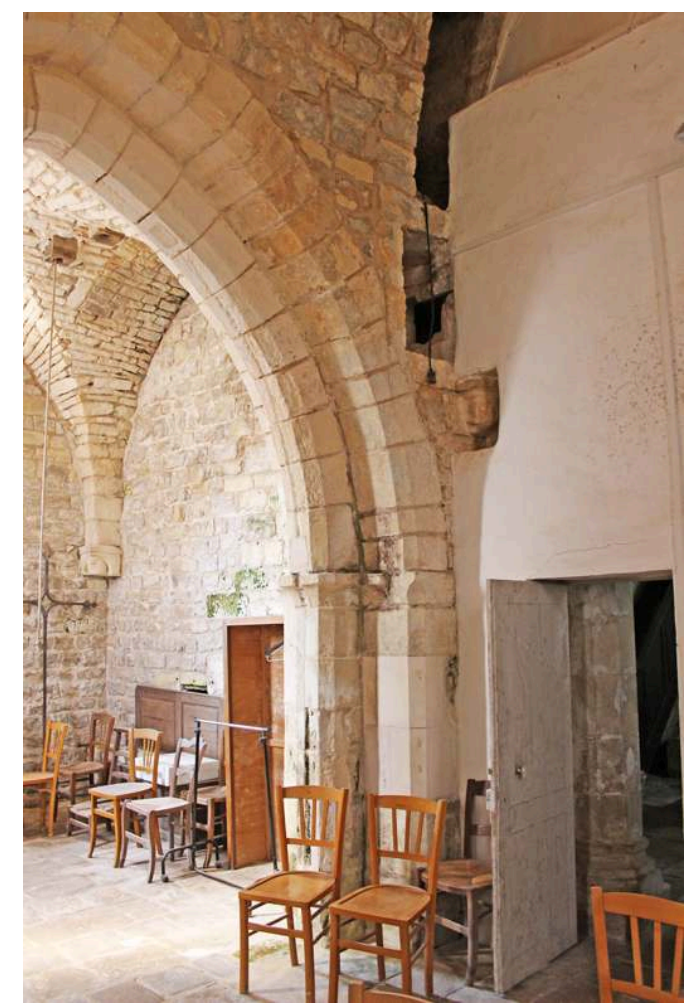

Une cavité aménagée dans la profondeur de l'arcade ouvrant sur l'abside, peinte, est dotée d'un système de fermeture. Seule une échelle devait permettre d'y accéder. La présence d'une source de lumière du côté de la nef rend toutefois difficile l'interprétation de cette cavité comme une cache.

\section{Changements de vocation et usages ponctuels}

Refermant cette parenthèse sur les "petits indices " susceptibles d'informer les fonctions des salles hautes et leurs relations aux motifs architecturaux contigus, il convient de revenir sur l'interprétation des dispositifs au prisme de la mise en pratique du rituel. La contribution d'Eduardo Carrero Santamaría à cette table ronde invite à une critique de l'approche unilatérale qui vise à mettre en rapport, comme c'est devenu la mode, les textes liturgiques avec les formes architecturales. M. Carrero prône une mise en perspective des données issues de ces textes avec l'anthropologie des rituels et de la religiosité populaire. L'étude des édifices doit en effet tenir compte des célébrations conditionnées par des contextes locaux particuliers: les processions et surtout les bénédictions. Ces pratiques étaient motivées par des facteurs propres au site. Elles pouvaient concerner le territoire entourant l'édifice ou encore le climat qu'il était nécessaire de conjurer tout au long de l'année et à des moments ponctuels comme les orages, afin d'éviter que la foudre ou la grêle ne dévastent les champs cultivés. La culture populaire a créé sa propre terminologie pour désigner les lieux d'où étaient conjurées les intempéries: on les appelait conjuratorios en Castille, esconjuradero en Aragon et comunidor en Catalogne. La terminologie médiévale était différente, utilisant le terme latin podicenus et surtout celui de reliquer, une claire référence à l'utilisation de 
reliques. En témoigne, notamment, le Libro del Subpriorado de la cathédrale de Saragosse rédigé par le chanoine Antonio Barberán vers 1470. Les communes de Saragosse étaient bénies à la Sainte-Croix de mai et le bras de saint Valero était monté dans le clocher chaque fois que les nuages menaçaient (fig. 8).

Fig. 8 - Saragosse, cathédrale, vue de la galerie des bénédictions (E. Carrero).

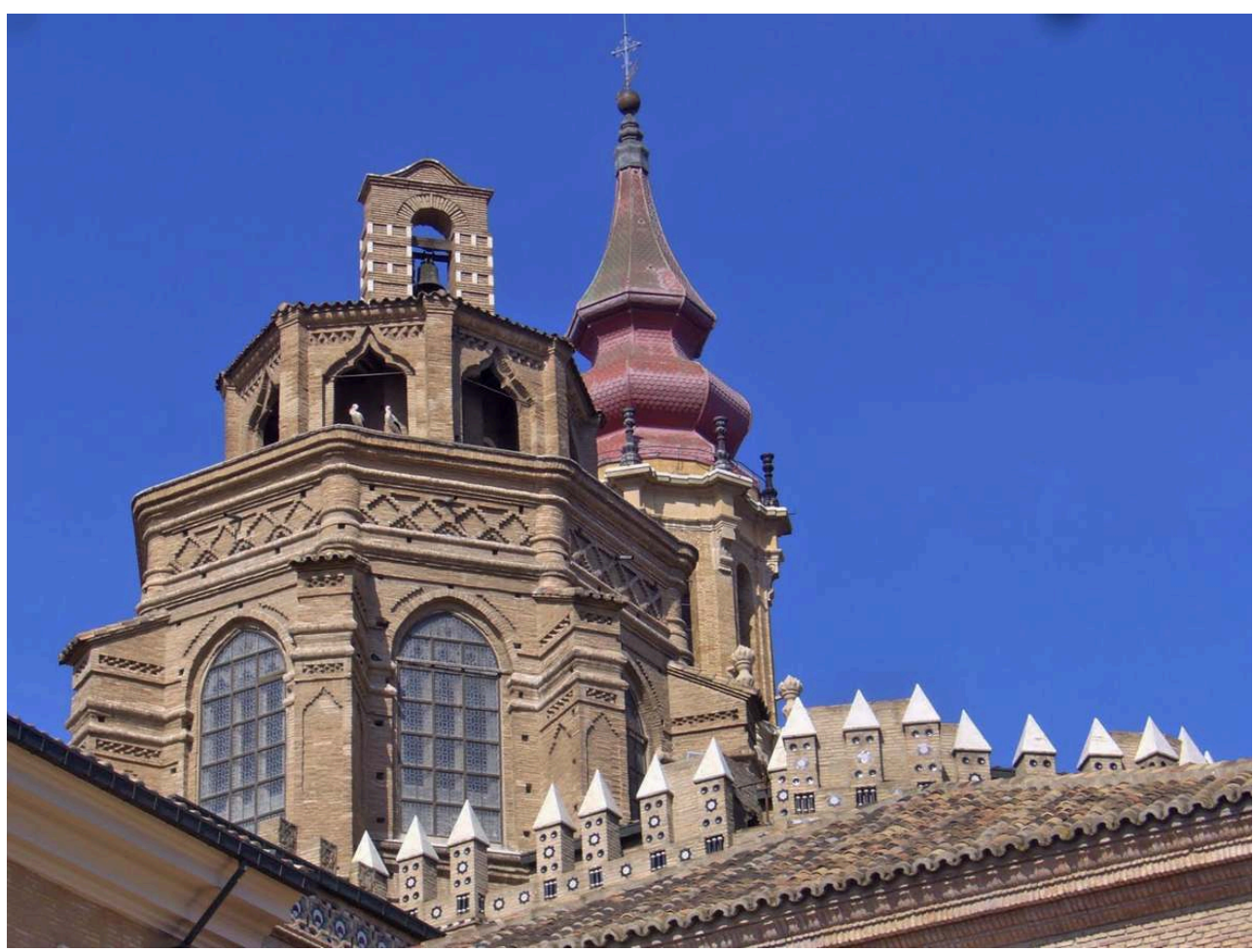

Pratiquant une démarche régressive, E. Carrero a remonté le cours du temps depuis le rituel pratiqué encore aujourd'hui, en mai, sur la croix du toit de la cathédrale de Barcelone appelée Pedró, jusqu'aux structures de l'époque romane tardive, comme la tour des Exorcismes de la cathédrale de Lleida (vers 1190). À San Benet de Bages monastère bénédictin de la région de Barcelone -, on édifia au XII ${ }^{\mathrm{e}}$ siècle une tour de croisée sans cloche avec un accès restreint depuis l'escalier qui communique avec les charpentes. Cette tour est parfaitement documentée comme lieu où l'on bénissait les champs qui entouraient le monastère et où l'on invoquait le beau temps. À partir de cet exemple, il devient possible d'inférer que des structures hautes de destination précise inconnue et dotées de systèmes de communication complexes avec le corps des édifices ont pu se prêter à cet usage, en particulier à Santa Cruz de la Serós - près de San Juan de la Peña (fig. 9) -, à l'église de Majones (également en Aragon), et à l'abbatiale San Martin de Frómista, où la structure d'où l'on conjurait le mauvais temps a été supprimée par la lourde restauration qu'a connue l'église, comme à la cathédrale de la Seu d'Urgell (fig. 10). 
Fig. 9 - Santa Cruz de la Serós, chevet de l'église vu depuis l'est (cl. E. Carrero).

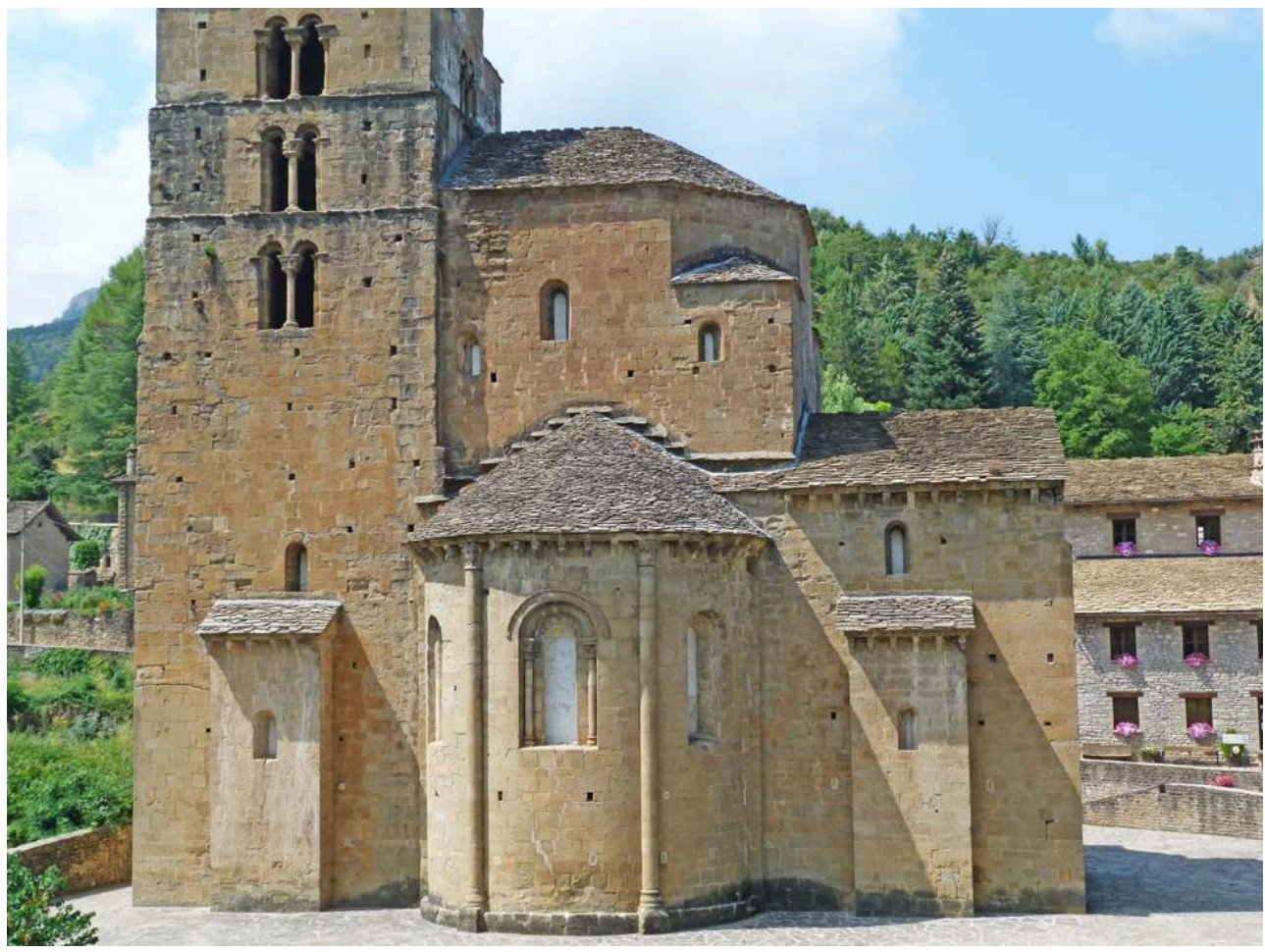

Fig. 10 - Frómista, San Martin, vue du chevet à la fin du XIXe siècle, selon F. J. Parcerisa.

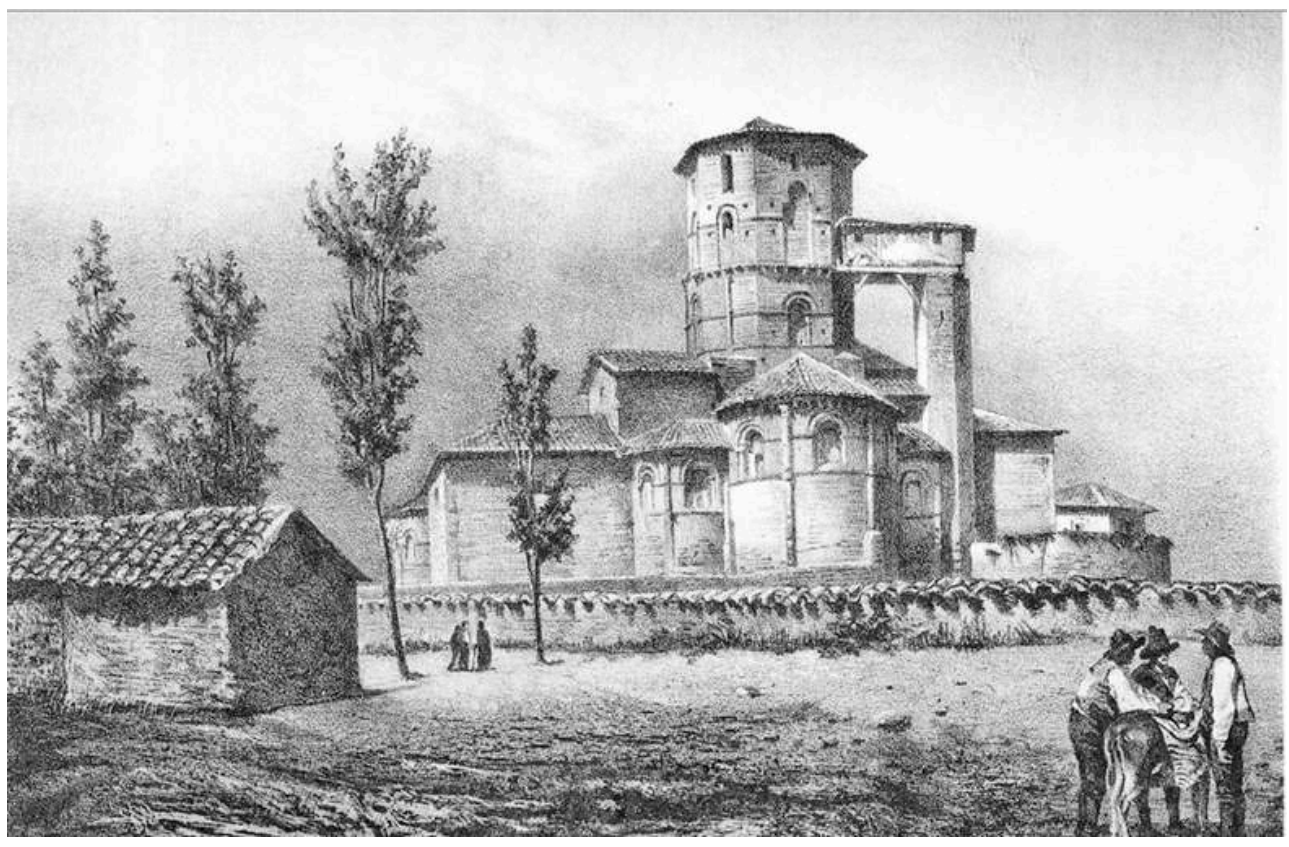

Une telle démarche implique une conception des fonctions des lieux qui soit perméable au changement dans l'histoire des édifices et tout au long de l'année liturgique : il faut admettre que des utilisations très différentes pouvaient coexister au sein d'un même espace. 


\section{Conclusion}

L'ensemble des dossiers abordés au cours de cette table ronde fait plus que jamais ressortir la nécessité de nouvelles études de cas, tant pour les édifices modestes que pour les plus fameux. Or, pour les espaces hauts comme pour toute question ayant trait aux choix formels, aux fonctions et aux usages, seul l'accroissement obstiné des faisceaux d'indices permet de fonder des hypothèses d'interprétation sérieuses. De grands édifices appellent une enquête approfondie : le clocher-porche de Saint-Hilairele-Grand à Poitiers attend une étude complète (fig. 11); la tour de Saint-Pierre de Lesterps nécessite des sondages archéologiques; la chapelle basse de Berzé-la-Ville a été négligée au profit de l'écrin pictural de la chapelle haute; la connaissance du premier état de l'avant-nef de Vézelay implique de fouiller le sol de l'avant-nef actuelle...

Fig. 11 - Poitiers, Saint-Hilaire-le-Grand, ancien clocher-porche vu depuis le nord (cl. C. AndraultSchmitt).

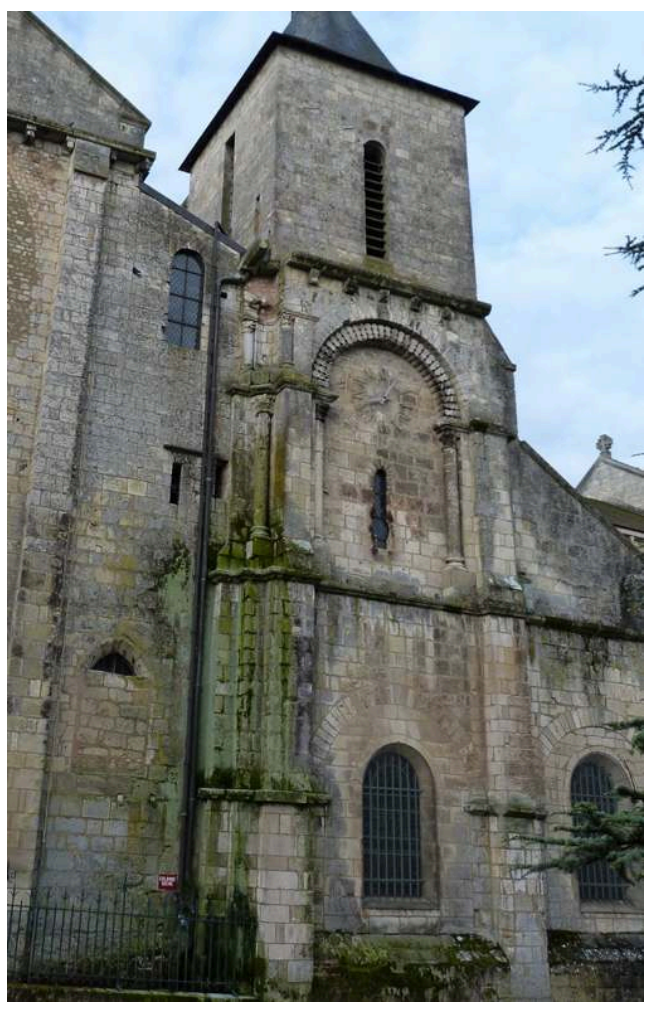

Peut-être l'arsenal des questions mérite-t-il encore d'être enrichi. Les communications prononcées lors de la table ronde ne pouvaient parvenir à donner une image exhaustive des motifs architecturaux concernés - la question des circulations hautes a été à peine entrevue, l'aborder de manière satisfaisante appelle un élargissement du sujet à l'architecture conventuelle - et il ne nous a été donné qu'un aperçu des usages ponctuels des espaces hauts ${ }^{31}$. L'éclatement géographique et l'absence de fil chronologique dans cet essai de synthèse ont peut-être décontenancé le lecteur. L'objectif n'était pas de brosser ni même d'esquisser un paysage monumental et rituel, mais bien de mettre en présence des objets et des motifs singuliers, de réfléchir pardelà les contrastes régionaux - sans accorder trop de place à la question des filiations -, de faire émerger un matériau vivant. Mettre sur la table des savoirs qui assument leur 
caractère provisoire, et surtout, confronter des cas singuliers - et leur cortège de problèmes - avec un large éventail d'hypothèses et de connaissances, afin de réveiller le débat. Surtout ne pas le clore.

Reçu : 16 décembre 2014 - Accepté : 16 mai 2015

\section{NOTES}

1. GERVASE DE CANTORBÉRY, Chronica Gervasii pars prima, in W. STUBBS (éd.), Historical Works of Gervase of Canterbury, Londres, 1879 (Rerum Britannicarum medii aevi scriptores, 73.1), p. 292-314. Traduction française Claude Andrault-Schmitt.

2. Nous signalons la parution récente de l'ouvrage de T. HUITSON, Stairway to Heaven : the Functions of Medieval Upper Spaces, Philadelphia, 2014, auquel les auteurs de ce texte n'ont pas eu accès avant la rédaction de cet article.

3. Nous sommes ici redevables à la bibliographie établie par C. КоscH, "Auswahlbibliographie zur Liturgie und Bildenden Kunst/Architektur im Mittelalter », in F. KOHLSCHEIN et P. wÜNSCHE (dir.), Heiliger Raum: Architektur, Kunst und Liturgie im mittelalterlichen Kathedralen und Stiftskirchen. Bamberger Liturgiewissenschaftliches Forschungskolloquium (4, 1995, Bamberg), Münster, 1998, p. 243--377 (Liturgiewissenschaftliche Quellen und Forschungen, 82). Ce n'est pas le lieu de reproduire ici l'abondante bibliographie des massifs occidentaux et Westwerke. Concernant les «chœurs-halles", voir les travaux récents: F. DHEIN, Die romanische Westchorhalle: Architekturhistorische Untersuchung eines Westbautypus und seiner Funktion, thèse de doctorat, Bonn, 2013 ; ID., " Le "chœur-halle occidental" à l'époque romane : un type d'édifice mosan étudié à la loupe ", in B. VAN DEN BOSSCHE (dir.), L'art mosan : Liège et son pays à l'époque romane du XI ${ }^{e}$ au XIII siècle, Liège, 2007, p. 100-106.

4. R. DORN, «Wo saßen die Stiftsdamen? Überlegungen zur Damenempore im Herforder Münster ", in Historisches Jahrbuch für den Kreis Herford (2001), p. 7-30; G. LEOPOLD, « Frauenemporen in Stifts- und Klosterkirchen des frühen Mittelalters im östlichen Sachsen », in R. SCHMITT, U. STEINECKE, et M. TITZE (dir.), «Es thvn iher viel fragen... ": Kunstgeschichte in Mitteldeutschland, Petersberg, 2001, p. 15-30; G. MUSCHIOL, «Liturgie und Klausur : zu den liturgischen Voraussetzungen von Nonnenemporen », in I. CRUSIUS (dir.), Studien zum Kanonissenstift, Göttingen, 2001, p.129-148 (Veröffentlichungen des Max-Planck-Instituts für Geschichte, 167; Studien zur Germania Sacra, 24).

5. B. BRENK, "Wer sitzt auf der Empore? ", in J. POESCHKE (dir.), Sinopien und Stuck im Westwerk der karolingischen Klosterkirche von Corvey, Münster, 2002, p. 71-86.

6. E. BADSTÜBNER, «Emporenkirche und Doppelkapelle: Vergleich eines Architekturmotivs in der frühmittelalterlichen Sakralbaukunst Transkaukasiens und des Abendlandes ", in Aachener Kunstblätter, 58 (1989-1990), p. 75-86; G. BINDING, K. WESSEL, « Doppelkapelle », in Lexikon des Mittelalters, Turnhout, 1986, t. 3, p. 1256-1257. 
7. Sur ce dernier point, voir C. MECKSEPER, Das Piano nobile: eine abendländische Raumkategorie, Hildesheim, 2012, p. 115-126 ; ID., «Oben und unten in der Architektur : zur Entstehung einer abendländischen Raumkategorie », in H. HIPP et E. SEIDL (dir.), Architektur als politische Kultur : philosophia practica, Berlin, 1996, p. 37-52.

8. Nous avons souhaité ne pas ouvrir la table ronde au public afin de préserver la dimension informelle ayant trait à la présentation d'études non abouties.

9. Le programme détaillé est à consulter: http://cescm.labo.univ-poitiers.fr/wpcontent/uploads/sites/49/2014/06/Affiche_Programme.pdf ; http://cescm.labo.univpoitiers.fr/wp-content/uploads/sites/49/2014/06/Affiche_Programme.pdf.

10. La chronique a été éditée par A. MARTINDALE, «The Romanesque Church of SaintBénigne at Dijon and MS 591 in the Bibliothèque Municipale ", in Journal of the British Archaeological Association, 25 (1962), p. 21-54 (ici p. 47-50), édition partiellement reproduite et traduite en français par Jacques Ménard dans C. M. MALONE, Saint-Bénigne de Dijon en l'an mil. Totius Galliae basilicis mirabilior: interprétation politique, liturgique et théologique, Turnhout, 2009 (Disciplina Monastica, 5), voir p. 290 pour les escaliers.

11. C. M. MALoNE, Saint-Bénigne..., ibid., fig. 18.

12. Carolyn M. Malone a mis en relation l'idée de progression vers Dieu par la lumière, exprimée dans un sermon de Jean de Fécamp (abbé de Saint-Bénigne entre 1052 et 1054), avec l'architecture de la rotonde qui possédait un grand puits de lumière centrale, une "hiérarchie spirituelle ascendante» (dédicaces des autels) et ces escaliers, qui, selon l'auteure, "contribuaient eux-mêmes à stimuler une réponse anagogique en correspondance avec la signification anagogique des dédicaces des autels situés sur les trois niveaux superposés de la rotonde » (C. M. MALONE, SaintBénigne..., ibid., p. 230, voir également p. 251).

13. T. BeLlA, La basilica di Sant'Ambrogio a Milano : l'opera inedita di Fernand De Dartein, Milan, 2013 ; EAD., «Dossiers des archives de Fernand de Dartein, IV. Tra le chiese di Milano, il caso di Sant'Ambrogio", in A. SEGAGNI MALACART et L. C. SCHIAVI (dir.), Architettura dell'XI secolo nell'Italia del Nord: storiografia e nuove ricerche. Convegno internazionale (Pavia, 8-9-10 aprile 2010), Pise, 2013, p. 25-33 et 300-311; ID., " À propos des armatures en bois dans les églises romanes de l'Italie du Nord : Saint-Ambroise et SaintCelse de Milan, Saint-Michel de Pavie », in Bulletin monumental, 170/4 (2012), p. 291-308 ; ID., « Dossiers d'archivio di Fernand de Dartein : nuovi contributi alla conoscenza della basilica di S. Ambrogio a Milano », in Arte medievale, 1 (2010-2011), p. 255-282 ; ID., « La basilica di S. Ambrogio a Milano nei dossiers d'archivio di Fernand de Dartein ", in Rivista dell'sstituto nazionale d'archeologia e storia dell'arte, 60 (2005), p. 235-273.

14. A. AMBROSIONI, Monaci e canonici all'ombra delle due torri, in M. L. GATTI PERER (dir.), La basilica di S. Ambrogio : il tempio ininterrotto, Milan 1995, t. 1, p. 241-251.

15. T. BellA, "Santa Maria del Tiglio a Gravedona: nuovi materiali sulla fabbrica romanica ", in G. GUARISCO, T. BELLA, M. LEONI, et D. MIRANDOLA (dir.), L'architettura romanica comasca e Fernand de Dartein. Storia, archeologia, tutela e restauri, Rome, à paraître.

16. Une chapelle surmontant le porche sud de Notre-Dame-la-Grande à Poitiers n'était sans doute accessible qu'au moyen d'une galerie de bois. C. ANDRAULT-SCHMITT, "L'architecture de l'église mariale au $\mathrm{XI}^{\mathrm{e}}$ siècle : une cohérence inattendue ", in M.- 
T. CAMUS et C. ANDRAULt-SCHMITT (dir.), Notre-Dame-la-Grande de Poitiers : l'œeuvre romane, Paris, 2002, p. 159-186, ici p. 185.

17. C. SAPIN, "Saint-Père (Yonne), l'ancienne église Saint-Pierre", Bulletin du Centre d'études médiévales d'Auxerre, 9 (2005), URL : http://cem.revues.org/656.

18. Sur le clocher de l'abbatiale Sainte-Marie de Zadar: E. HILJE, "Combinations of Romanesque and Gothic Forms in the Architecture of Zadar", Hortus Artium Medievalium, 2 (1996), p. 65-76, ici p. 71-73; sur la tour de l'église Saint-Sauveur à la source de la Cetina : M. JURKović, «L'Église et l'état en Croatie au IX siècle : le problème du massif occidental carolingien », in Hortus Artium Medievalium, 3 (1997), p. 23-40, ici p. 23-24.

19. C. SAPIN, « D'Auxerre à Cluny, le dossier archéologique des premières avants-nefs et galilées ", in C. SAPIN (dir.), Avant-nefs et espaces d'accueil dans l'église (entre le IVe et le XII siècle), Paris, 2002, p. 398-413, ici p. 399.

20. C. SAPIN, "Saint-Marcel-lès-Chalon (Saône-et-Loire), église Saint-Marcel», Bulletin du Centre d'études médiévales d'Auxerre, 10 (2006), URL : http://cem.revues.org/455.

21. K. KRÜGER, Die romanischen Westbauten in Burgund und Cluny: Untersuchungen zur Funktion einer Bauform, Berlin, 2003 ; « Architecture and Liturgical Practice : The Cluniac Galilea », in N. нІзсоск (dir.), The White Mantle of Churches: Architecture, Liturgy, and Art around the Millennium, Turnhout, 2003, p. 136-159 (International medieval research, 10).

22. Les résultats de cette étude ont fait l'objet d'une publication récente : S. BÜTTNER, «La mise en œuvre de la façade et du grand portail de la nef de Vézelay: nouvelles données archéologiques", in Le portail roman (XI-XII siècles): nouvelles approches, nouvelles perspectives. Actes des $45^{e}$ Journées romanes de Cuxa, Abbaye Saint-Michel de Cuxa (Codalet-Prades), 2014, p. 145-156 (Les cahiers de Saint-Michel-de-Cuxa, 45).

23. Il ne s'agit pas pour autant d'un phénomène général: des salles hautes sont aménagées dans les clochers de Saint-Yrieix et Meymac dans le second quart du $\mathrm{XII}{ }^{\mathrm{e}}$ siècle.

24. É. SPARHUBERT, Les commandes artistiques des chapitres de chanoines séculiers et leurs enjeux. Édifier et célébrer à Saint-Junien (XI -XIII siècles), thèse de doctorat, université de Poitiers, 2008, p. 284 sqq.

25. C'est ainsi que sont interprétées les salles hautes au-dessus des sacristies dans l'ouvrage de J. BURTON et J. KERR (dir.), The Cistercians in the Middle Ages, Woodbridge/ Rochester, 2011.

26. F. GANDOLFo, «Le cattedrali siciliane », in A. C. QuinTAVAlle (dir.), Medioevo : l'Europa delle cattedrali, Milan, 2007, p. 191-207 (I Convegni di Parma, 9).

27. Les fruits de ce travail seront publiés prochainement : С. козсн, Hochmittelalterliche Sakralbauten in Naumburg, Schulpforte und Freyung an der Unstrut Architektur und Liturgie bis 1300, Ratisbonne, à paraître en septembre 2015 (Grosse Kunstführer, 268).

28. La construction des tours remonte aux années 1220 , antérieurement à l'aménagement de l'abside occidentale.

29. M. L. CASATI, « Note sulle fasi costruttive medioevali delle chiese di San Vincenzo e di Santa Maria del Tiglio ", in D. PEScARMona (dir.), La croce lignea di Gravedona : storia e restauro del Crocifisso romanico di Santa Maria del Tiglio, Milan, 2002, p. 31-43, ici p. 35 ; S. LOMARTIRE, "L'organisation des avant-corps occidentaux: à propos de quelques 
exemples de l'Italie du Nord au Moyen Âge ", in C. SAPIN (dir.), Avant-nefs et espaces d'accueil..., op. cit., p. 351-371; M. BELLONI ZECCHINELLI, « Le origini della "romanica" Santa Maria del Tiglio di Gravedona », in P. SANPAOLESI (dir.), Il Romanico. Atti del seminario di studi, Villa Monastero di Varenna (8-16 settembre 1973), Milan, 1975, p. 341-369.

30. Voir l'étude de l'église dans C. ARNAUD, Les églises de l'ancien diocèse d'Auxerre: du milieu du Xl siècle au début du XIII siècle, Auxerre, 2009.

31. Il était difficile de présenter dans cette synthèse l'intégralité des dossiers traités lors de la table ronde. Mentionnons encore les chevets à plusieurs niveaux de SaintGermain d'Auxerre et de Saint-Pierre de Flavigny, l'église Notre-Dame-sous-Terre du Mont-Saint-Michel ( $\mathrm{x}^{\mathrm{e}}$ siècle) avec ses tribunes situées au-dessus des absides (présentations : Christian Sapin), ainsi que l'étage de la tour Sainte-Croix (xII siècle) de la priorale de la Charité-sur-Loire (Nièvre) et ses aménagements venus en doublage de la maçonnerie d'origine (présentation : Fabrice Henrion).

\section{ABSTRACTS}

The study of upper spaces in medieval churches calls for a multidisciplinary approach monumental archaeology, art history, history of liturgy and of medieval text. This paper argues that a comprehensive study of the aforementioned spaces is indeed essential in order to obtain a better understanding of medieval places of worship and the society that built them. The arguments it brings forward are those expressed by a recent panel whose intention was to raise questions, compile issues and establish points of view rather than build interpretative models. The result of this is an array of observations on French, Italian, Spanish, German and AngloNorman buildings, dating from the 10th to the 13th centuries. The examples offered by the contributors of this panel are not representative of any trend; rather they deal with problems in interpreting space, layouts and openings according to an intended public or use (ritual or not). The particular attention given to the regions of Aquitaine and Burgundy reveals a dynamic transformation of patterns which neatly summarizes the subject of the panel as it illustrates the plural and changing purposes of upper spaces in medieval churches and thus confirms the need to study them accordingly.

L'étude des espaces hauts des églises médiévales implique l'intégration de spécialités multiples l'archéologie du bâti, l'histoire de l'art, la liturgie, l'histoire des textes - dans un effort de réflexion commun. Le présent article entend montrer que cette démarche intégratrice est efficace et fructueuse dans l'optique d'une étude renouvelée et globale de ces espaces. Or, une telle étude apparaît nécessaire pour une meilleure connaissance des lieux de culte ainsi que de la société qui les a produits et utilisés. Ainsi cet article rend-il compte des travaux d'une table ronde organisée dans le souci de faire émerger des questions, de sérier des problèmes, de proposer des points de vue plutôt que des modèles d'interprétation. Il en résulte une mosaïque d'observations sur des édifices français, italiens, espagnols, allemands, anglo-normands, allant du xi siècle au $\mathrm{XIII}^{\mathrm{e}}$ siècle. Les exemples choisis par les contributeurs ne se veulent en rien représentatifs de tendances générales; ils sont en revanche révélateurs des problèmes d'interprétation des espaces, aménagements et systèmes d'ouvertures en fonction de leur destination (à des 
personnes) et de leurs usages (rituels ou non). L'attention particulière portée sur les régions Aquitaine et Bourgogne fait apparaître une dynamique de transformation des motifs qui caractérise le sujet de cette synthèse aussi bien que la nécessité de penser les fonctions de manière plurielle et changeante.

\section{INDEX}

Geographical index: France, Angleterre, Allemagne, Italie, Espagne

Mots-clés: architecture, archéologie, liturgie, église romane, hauteur, tribune, tour, chapelle, galilée, avant-nef, escalier, galerie

\section{AUTHORS}

\section{SÉBASTIEN BIAY}

Docteur en histoire de l'art médiéval, ingénieur de recherche

Programme ANR Musiconis -Centre d'études supérieures de civilisation médiévale (UMR 7302, université de Poitiers/CNRS)

\section{ANNICK GAGNÉ}

Doctorante en histoire médiévale

Centre d'études supérieures de civilisation médiévale (UMR 7302, université de Poitiers/CNRS) et Département des sciences historiques (université de Laval, Canada) 